\title{
Tuberculosis resistance protein TOLLIP controls immune pathology by resolving the integrated stress response in alveolar macrophages.
}

Sambasivan Venkatasubramanian

University of Washington

Courtney Plumlee

Seattle Children's Research Institute

Kim Dill-McFarland

University of Washington

Gemma Pearson

University of Michigan

Sara Cohen

Seattle Children's Research Institute

Anne Lietzke

University of Michigan

Amanda Pacheco

University of Michigan

Sarah Hinderstein

University of Washington

Scott Soleimanpour

University of Michigan

Matthew Altman

University of Washington

Kevin Urdahl

Seattle Children's Research Institute

Javeed Shah ( $\sim$ jashah@uw.edu )

University of Washington https://orcid.org/0000-0002-2997-7988

Article

Keywords: TOLLIP, tuberculosis, EIF2, alveolar macrophages, integrated stress response, mycolic acid

Posted Date: February 23rd, 2022

DOI: https://doi.org/10.21203/rs.3.rs-1363035/v1 
License: (c) (i) This work is licensed under a Creative Commons Attribution 4.0 International License. Read Full License 


\section{Abstract}

A functional polymorphism responsible for TOLLIP deficiency is associated with increased tuberculosis risk. However, TOLLIP's role in TB pathogenesis is unknown. We found that Tollip ${ }^{-/-}$mice develop severe Mycobacterium tuberculosis (Mtb) disease, characterized by lipid-laden foamy macrophages. This phenotype was intrinsic to alveolar macrophages (AM), as Tollip ${ }^{-/-}$AM from mixed bone marrow chimeras selectively accumulated lipid and harbored Mtb. Global gene expression analysis of Mtbinfected, Tollip ${ }^{-/-}$AM demonstrated increased EIF2 signaling, a key regulator of the integrated stress response (ISR) to variable environmental conditions. The ISR and foam cell formation were linked, as Mtb lipid mycolic acid incubation with Tollip ${ }^{-/-}$macrophages led to lipid accumulation, ISR responses, and increased Mtb replication. Correspondingly, ISRIB, a small-molecule ISR inhibitor, restored Mtb control in Tollip $^{-/-}$mice and substantially improved Mtb outcomes in resistant C57BL/6 mice. Targeting the ISR broadly improves Mtb control and immunopathology, offering a promising target for host-directed tuberculosis therapy.

\section{Main}

A common, functional promoter variant in Toll-Interacting Protein (TOLLIP) is associated with TOLLIP deficiency and increased risk for Mycobacterium tuberculosis (Mtb) disease ${ }^{1,2,3}$. TOLLIP is a ubiquitinbinding protein, expressed in all tissues, that organizes the transport and delivery of endosomal cargo 4,5 , ${ }^{6}$. Its cargo include intracellular lipids ${ }^{7}$, insoluble protein aggregates ${ }^{8,9}$, cell surface and endosomal signaling receptors ${ }^{10}$, and mitochondria ${ }^{11,12}$, but its complex and varied role in endosomal transport remains incompletely defined. Via this activity, TOLLIP diminishes TLR2, TLR4, and IL1-R signaling, stabilizes Type I interferon secretion via the endosomal immune receptor STING, and significantly reduces immune pathology during prolonged LPS stimulation ${ }^{10,13,14,15,16}$. TOLLIP influences pathogenesis of several chronic conditions, including neurodegenerative disease ${ }^{8,9}$, pulmonary fibrosis ${ }^{17}$, stroke $^{18}$, and myocardial infarction ${ }^{19}$. Thus, TOLLIP regulates a plethora of diverse cellular functions and pathological conditions, but how it influences Mtb pathogenesis is unknown.

Macrophages are good candidate mediators for TB susceptibility in TOLLIP-deficient individuals. Macrophages are the primary intracellular niche for Mtb in vivo, and the relative activation state of the macrophage determines whether this niche is permissive or restrictive to Mtb replication. TOLLIP's diverse functions significantly overlap with critical host defense pathways responsible for Mtb control. TLR2, TLR4, IL-1R, and STING participate in Mtb recognition by innate immune cells and contribute to Mtb host defense ${ }^{20,21,22}$. Complex ER endosomal sorting restricts intracellular Mtb growth ${ }^{23}$, and oxidative damage from senescent mitochondria induces Mtb spread in macrophages ${ }^{24,25}$. Accumulation of lipids and insoluble protein aggregates within macrophages leads to foamy macrophage formation and Mtb persistence in vivo $26,27,28$. Therefore, understanding how TOLLIP restricts Mtb growth within macrophages may provide insight into essential Mtb host response pathways, especially during chronic infection. To better understand how TOLLIP influences Mtb-macrophage interactions in vivo, we 
conducted a series of experiments in genetically modified mice to identify the lung-resident macrophage subsets most dependent on TOLLIP for Mtb host defense and the specific cellular mechanisms responsible for this phenotype.

\section{Results}

\section{TOLLIP is required to control Mtb infection}

Human TOLLIP-deficient macrophages developed hyperinflammatory cytokine responses after TLR stimulation or live Mtb infection ${ }^{1,2,3}$. We hypothesized that murine TOLLIP deficiency might be linked to similarly dysregulated cytokine responses. To test this, we evaluated cytokine responses in Tollip ${ }^{-/-}$ macrophages after TLR stimulation or Mtb infection. Tollip ${ }^{-/-}$peritoneal macrophages (PEM) secreted more TNF but less IL-10 after LPS (TLR4 ligand), PAM3 (TLR2/1 ligand), or Mtb whole cell lysate stimulation (Figure S1A-B), and more TNF and IL-1b, but less IL-10 after overnight Mtb infection (Figure S1C-E; H37Rv strain, $\mathrm{MOI} 2.5)$. These findings are consistent with our prior studies and suggest that Tollip-mediated regulation of macrophage function is similar in humans and mice ${ }^{3}$.

TOLLIP maintains immune balance in macrophages after prolonged stimulus, and hyperinflammatory immune activation is associated with worsened Mtb outcomes ${ }^{29}$. Therefore, we hypothesized that Tollip $^{-/-}$mice would develop worsened Mtb disease. We infected Tollip ${ }^{-/-}$mice and littermate controls (B6) with $~ 50$ colony forming units (CFU) of Mtb H37Rv via aerosol and monitored weight, bacterial burden, and survival over time (Fig. 1A). Lung bacterial burdens were diminished in Tollip ${ }^{-/-}$mice 7, 14 (Fig. 1B) and 28 days (Fig. 1C) post-infection but developed increased bacillary burden 56 days postinfection and onward. By 180 days post-infection, lung bacterial burdens were increased ten-fold in Tollip $^{-/-}$mice (Fig. 1C). In the spleen, Mtb dissemination was delayed in Tollip ${ }^{-/-}$mice, as Mtb was not detected in 2/5 spleens 14 days post-infection (Fig. 1D). We detected fewer Mtb CFU in the spleens of Tollip $^{-/-}$mice 28 days post-infection but increased Mtb CFU 56 days post-infection and onward (Fig. 1E). Tollip $^{-/-}$mice exhibited greater weight loss (Fig. 1F) and met criteria for euthanasia before B6 controls (Fig. 1G). Histopathologic analysis of lung sections revealed distinct patterns in Tollip $^{-/-}$mice 56 days after infection, displaying increased numbers of inflammatory infiltrating cells with finely fibrillary cytoplasmic appearance, consistent with lipid-laden "foamy" macrophages (Fig. 1H). Taken together, Tollip ${ }^{-/-}$mice developed worsened Mtb disease and large numbers of foamy macrophages during prolonged infection.

TOLLIP mRNA and protein expression is ubiquitous in both hematopoietic and non-hematholpoietic, cells ${ }^{5,30}$. To assess the relative contribution of each cell type to Mtb immunity, we generated bonemarrow chimeric mice and infected them with aerosolized Mtb. Bacterial burdens were determined 60 days after infection (Fig. 1I). Tollip ${ }^{-/-}$bone marrow $->$Tollip $^{-/-}$mice exhibited higher lung bacterial burdens that B6 -> B6 controls. Importantly, Tollip ${ }^{-/-} \rightarrow$ B6 chimeras developed elevated bacterial burden, 
while bacillary load in B6 $\rightarrow$ Tollip ${ }^{-/-}$was not significantly different than B6 -> B6 controls (Fig. 1J). Therefore, TOLLIP influences Mtb protection via expression in radiosensitive hematopoietic cells.

We also measured the contribution of TOLLIP to Mtb host defense in the absence of mature B and T cells. We compared bacterial burden of Tollip ${ }^{+/}$, Tollip $^{-/-}, \operatorname{Rag}^{1^{-/}}$, and Rag $^{-/-}$Tollip $^{-/-}$mice 28 days after aerosol infection with Mtb. Rag $1^{-/-}$Tollip $^{-/-}$mice demonstrated significantly increased Mtb CFU in their lungs (2.2x higher CFU) compared to Rag $1^{-/-}$mice (Fig. $\left.1 \mathrm{~K}\right)$. These data demonstrate that TOLLIP is required for an optimal innate immune response against $\mathrm{Mtb}$, in the absence of $\mathrm{T}$ and $\mathrm{B}$ cell responses.

\section{Tollip $^{-/-}$alveolar macrophages selectively harbor increased Mtb}

Lung-resident macrophage subset populations demonstrate distinct, genetically regulated functional characteristics that influence responses to $\mathrm{Mtb}^{31,32}$. Only some $\mathrm{Tollip}^{-/-}$macrophages developed a foamy phenotype, and TOLLIP was required for myeloid cell Mtb control. Therefore, we considered whether TOLLIP's effects were selective to specific macrophage subpopulations. Tollip ${ }^{-/-}$and B6 mice had similar numbers of alveolar macrophages (AM; CD11c + SiglecF+), monocyte-derived macrophages (MDM; SiglecF-CD11b + CD11c + MHCII+), interstitial macrophages (IM; SiglecF-CD11b + CD11c-MHCII), neutrophils (PMN; SiglecF-CD11b + Ly6G+), and dendritic cells (DC; SiglecF-CD11b-CD11c + MHCII+) after 28 or 56 days of infection with Mtb expressing an mCherry fluorescent reporter (Mtb-mCherry; Fig. 2A-B, gating in Figure S2) ${ }^{33,34}$. In Tollip ${ }^{-/-}$mice, fewer AM and PMN were infected with Mtb-mCherry 28 days postinfection (Fig. 2C), but after 56 days, a significantly greater proportion of AM and MDM were Mtbinfected (Fig. 2D). The frequency of Mtb-infected AM, but not MDM or PMN, was significantly increased in Rag $^{-/-}$Tollip $^{-/-}$mice, compared with Rag $1^{-/-}$control mice, 28 days after infection (Fig. 2E). These data suggest that Tollip $^{-/-}$AM may be selectively susceptible to Mtb, and that $\mathrm{T}$ and $\mathrm{B}$ cells delay, but do not eliminate, this effect.

We hypothesized that intrinsic TOLLIP activity led to selective effects on AM. To test this, we generated mixed bone marrow chimeric mice, which permit evaluation of TOLLIP function at the single cell level. B6.SJL-Ptprca Pepcb/BoyJ (CD45.1) mice were lethally irradiated and bone marrow was reconstituted with a 1:1 mix of bone marrow from Tollip ${ }^{-/-}(\mathrm{CD} 45.2+)$ and B6 (CD45.1 + CD45.2+) mice, followed by 10 weeks of rest (Fig. 2F). After confirming equal proportions of immune cells from each lineage (Fig. 2G), we infected chimeras with Mtb-mCherry and compared the proportion of Mtb-infected myeloid cell subsets by genotype (gating strategy depicted in Figure S3A-D). AM were the primary cell infected 14 days post-infection, with increasing proportions of PMN and MDM becoming infected over time, consistent with prior findings (Fig. $2 \mathrm{H})^{34} .14$ days post infection, proportionally fewer Tollip $^{-/-}$AM, but not other subpopulations, were Mtb-infected (Fig. 2l). By contrast, proportionally more Tollip ${ }^{-/-}$AM were Mtb infected and intracellular Mtb MFI was increased, suggesting greater intracellular Mtb burden, but other Tollip ${ }^{-/-}$myeloid subsets did not demonstrate relative Mtb accumulation (Fig. 2J-K). Tollip ${ }^{-/-} \mathrm{AM}$ demonstrated increased NOS2 + frequency (Figure S3E) with increased MFI (Figure S3F), confirming that 
Mtb persistence occurred in the setting of hyperinflammation. Therefore, absence of Tollip in AM leads to selectively increased Mtb burden during prolonged infection.

\section{Tollip $^{-/-}$AM develop increased EIF2 signaling after prolonged Mtb infection}

We next sought to identify the gene expression networks in AM that were associated with prolonged Mtb infection. We compared the global transcriptional networks of sorted, Mtb-infected and bystander B6 and Tollip $^{-/-}$AM from mixed bone marrow chimeric mice 28 days after Mtb infection (sorting strategy is shown in Figure S4). We identified 194 differentially expressed genes (DEG) between B6 and Tollip ${ }^{-/-}$ Mtb-infected AM (Fig. 3A; FDR < 0.05; gene names in Dataset S1) and 157 DEGs between B6 and Tollip ${ }^{-/-}$ Mtb-uninfected "bystander" AM, and with a more liberal FDR < 0.3, we identified 2599 DEGs between Mtbinfected AM and 2042 DEGs from bystander AM (Fig. 3B; Dataset S1). We performed Ingenuity Causal Network Analysis comparing DEGs from Mtb-infected B6 and Tollip ${ }^{-/-}$AM to identify functional nodes that were associated with their increased susceptibility to Mtb infection ${ }^{35}$. The top node discovered in Tollip ${ }^{-/-}$AM was "Increased EIF2 Signaling" (Fig. 3C).

EIF2 signaling is activated via phosphorylation at Ser51 of EIF2A, which triggers the integrated stress response (ISR), a set of cellular adaptations to harsh environmental or cellular conditions ${ }^{36}$. We validated our transcriptional observations by measuring phosphorylated EIF2A (pEIF2) in Mtb-infected lungs by immunohistochemistry. We found increased pEIF2 in the lungs of Tollip ${ }^{-/-}$mice 56 days after aerosol Mtb infection (Fig. 3D-E) ${ }^{37}$ with no difference in total EIF2A expression (Fig. 3F-G). To summarize, Tollip ${ }^{-1}$ - AM in mixed chimeric mice demonstrated increased EIF2 signaling 28 days after infection via transcriptional and protein analysis, which correlated with increased bacterial burden.

\section{TOLLIP and EIF2 kinase expression is increased in caseous human TB granulomas}

A defining characteristic of human TB granulomas is the presence of "caseum," a lipid-rich center of necrosis that is surrounded by foam cells and other inflammatory cells induced by Mtb infection 38,39 . Because we noted that TOLLIP acts selectively in AM to influence EIF2 activation, we hypothesized that TOLLIP and EIF2 kinase expression are increased in human caseous granulomas. EIF2 is phosphorylated by one of four upstream kinases - EIF2AK1 (HRI), EIF2AK2 (PKR), EIF2AK3 (PERK), and EIF2AK4 $(G C N 2)^{40}$. We evaluated the gene expression of TOLLIP, EIF2A (EIF2S1), and EIF2 kinases in human caseous granulomas compared with samples from healthy areas of the same lung, using a publicly available dataset ${ }^{41}$. EIF2AK1, EIF2AK2, and EIF2AK3 demonstrated significantly increased expression in caseous granulomas, but not EIF2S1 (Fig. 4A-D). EIF2AK4 and TOLLIP expression were increased (Fig. 4E-F; FDR $=0.25$ and 0.08 , respectively) but less strongly than other EIF2 kinases. In contrast to our observations in granulomas, TOLLIP and EIF2 kinase expression was not changed in the peripheral blood of individuals with active or latent TB (Figure S5A-D) ${ }^{42}$. Together, EIF2 kinases and TOLLIP are selectively enriched in the TB granuloma, providing a tissue-specific role for these genes during human TB disease. 
Mtb cell wall lipid mycolic acid induces lipid accumulation and increases Mtb replication in Tollip ${ }^{-/-}$ macrophages

Next, we sought to assess how TOLLIP deficiency shapes ISR activation in Mtb-infected AM. TOLLIP prevents lipid droplet delivery to lysosomes ${ }^{7}$, excess lipid induces the $\mathrm{ISR}^{43}$, and lipid accumulation may contribute to myeloid cell dysfunction via cellular stress pathways ${ }^{44,45}$. Therefore, we considered the possibility that lipid accumulation in Tollip ${ }^{-/-}$AM contributes to Mtb spread by inducing the ISR via pEIF2 ${ }^{46}$. To understand this pathway better, we evaluated two known mechanisms for inducing lipid accumulation and foam cell formation in Mtb-infected macrophages -- Type I interferon and the Mtb cell wall lipid mycolic acid (MA $)^{47,48}$. In Tollip ${ }^{-/-}$mice, Ifnb gene expression was diminished in Mtb-infected lung tissue 60 days after infection (Fig. 5A) ${ }^{15}$. Therefore, we evaluated how TOLLIP influenced MAinduced lipid accumulation in macrophages. We confirmed the capacity of $\mathrm{Tollip}^{-/-}$bone marrow-derived macrophages to perform bulk macroautophagy, a major lipid clearance pathway ${ }^{43,49,50}$. In these cells, we measured protein levels of LC3II and p62 after treatment with Bafilomycin A (BafA) to prevent autophagosome/lysosome fusion ${ }^{51}$. Tollip did not significantly affect levels of LC3II, nor the induction of LC3II by BafA treatment (Figures S6A-B). Levels of the adaptor protein p62, which is cleared during autophagy, were also similar (Figures S6C). Therefore, TOLLIP is dispensable for bulk autophagic flux in bone marrow-derived macrophages.

Next, we evaluated lipid accumulation after MA treatment. Tollip ${ }^{-/-}$PEM selectively accumulated lipid droplets after treatment with a mix of three MA isoforms found in Mtb (a-MA, methoxy-MA, and keto-MA) for 72 hours (Fig. 5B-D) $27,52,53$. MA-treated Tollip ${ }^{-/-}$PEM had increased Mtb replication, measured by luminescence, after infection with Mtb H37Rv expressing the luxCDABE luminescent reporter from Vibrio harleyi (Mtb-lux, MOI 1), which closely correlates with bacterial CFU (Fig. 5E) ${ }^{2}$. We observed similar phenotypes in vivo. In mixed bone marrow chimeric mice, Tollip ${ }^{-/-} \mathrm{AM}$, but not MDM, accumulated lipid 28 days after Mtb infection (Fig. 5F-G, gating in Figure S4). Taken together, MA is sufficient to induce lipid accumulation and promote Mtb replication in Tollip ${ }^{-/-}$macrophages, and we observed selective lipid accumulation in Tollip ${ }^{-/-}$AM that corresponded with increased Mtb burden in mixed bone marrow chimeras.

\section{EIF2 inhibition with ISRIB controls Mtb replication and improves Mtb outcomes}

After determining that lipid accumulation was associated with Mtb replication in Tollip ${ }^{-/-}$macrophages, we evaluated the effect of MA on ISR activation. MA treatment of Mtb-infected, Tollip ${ }^{-/-}$PEM led to significant increases in Ern1 (Figure S7A), Eif2ak3 (Figure S7B), and Atf6 (Figure S7C), which maintain immune function during lipid excess and activate the ISR via Eif2ak $3^{44,46,54}$. Because MA is linked to ISR activation, we evaluated the influence of the ISR on Mtb replication directly, by treating PEM with the EIF2 activator raphin-1 or the EIF2 inhibitor ISRIB ${ }^{55,56}$. Neither raphin-1 $(10 \mu \mathrm{M})$ or ISRIB $(5 \mathrm{nM})$ treatment induced cell death in macrophages or altered Mtb replication in broth culture (data not shown). Raphin-1 
treatment increased Mtb replication in PEM (Fig. 6A) while ISRIB decreased Mtb replication in MA-treated, Tollip ${ }^{-/-}$PEM (Fig. 6B). Overall, these data confirm that ISR activation permits Mtb replication independently of lipid accumulation, and that MA-induced Mtb replication is mediated through ISR activation.

Next, we tested the effectiveness of ISRIB on Mtb control in vivo. We infected mice with Mtb via aerosol, and after allowing 15 days for Mtb growth, treated daily with vehicle control, ISRIB $(1 \mathrm{mg} / \mathrm{kg}$ intraperitoneally), or raphin- 1 ( $1 \mathrm{mg} / \mathrm{kg}$ intraperitoneally). 56 days post infection, Mtb CFU were measured from lungs and spleen, and one lung lobe was preserved for pathology analysis (Fig. 6C). ISRIB treatment restored Mtb control in the lungs and spleens of Tollip ${ }^{-/-}$mice (Fig. 6D-E). Moreover, ISRIB significantly reduced overall bacterial burden in resistant B6 control mice (Fig. 6D-E). Treatment of B6 mice with raphin-1 increased bacterial burden in both lung and spleen (Fig. 6F-6G). In pathology analysis, ISRIB significantly reduced lung cellularity in the hyperinflammatory Tollip ${ }^{-/-}$mouse model, but neither ISRIB or raphin-1 significantly altered lung cellularity in $\mathrm{B} 6$ mice (Fig. 6H-6l) ${ }^{9,57}$. We detected fewer cells and increased hematoxylin staining in raphin-1 treated, Tollip ${ }^{-/-}$mice, consistent with cellular necrosis, which is a risk factor for Mtb spread ${ }^{29}$. In summary, ISRIB treatment rescued the Mtb susceptibility phenotype in Tollip $^{-/-}$mice and significantly improved Mtb control in B6 mice.

\section{Discussion}

TOLLIP deficiency in mice was associated with enhanced Mtb control in the early stages of Mtb infection, but ultimately resulted in exacerbated disease. The worsened immunopathology in Tollip ${ }^{-/-}$mice was associated with an abundance of lipid-laden foamy macrophages, and a failure to control bacterial burdens during chronic infection. AM were shown to be the primary cellular drivers of this phenotype, as AM TOLLIP-deficiency resulted in intracellular lipid accumulation, which triggered an EIF2-mediated stress response. EIF2 inhibition resulted in improved pathology and Mtb control, providing a mechanistic link between TOLLIP deficiency and Mtb disease, and identifying this pathway as a potential target for host-directed therapy.

A schematic of our proposed model is shown in Fig. 6J. Tollip ${ }^{-/-}$AM accumulate lipids intracellularly during Mtb infection due to selectively impaired homeostatic lipid clearance of Mtb lipids. Mtb cell wallderived lipids, such as MA, induce ISR activation, which contributes to increased intracellular Mtb replication, as EIF2 inhibition via ISRIB restores macrophage host defense in Tollip ${ }^{-/-}$mice. However, in TOLLIP-deficiency, AM provide a reserve niche for Mtb persistence during chronic infection.

It is interesting that AM-intrinsic TOLLIP-deficiency only contributes to exacerbated disease during prolonged infection, but not in the first few weeks after aerosol infection. In previous in vitro experiments, we observed improved Mtb control in Tollip ${ }^{-/-}$macrophages after 48 hours, and improved control of $L$. pneumophila infection, an acute intracellular bacterial infection, in Tollip ${ }^{-/-}$mice. These data are consistent with an enhanced and beneficial innate immune response during acute infections ${ }^{2,9}$. However, 
Tollip $^{-/-}$AM developed lipid accumulation that corresponded with impaired Mtb control that corresponds with lipid accumulation during the chronic phase of Mtb infection. Therefore, TOLLIP may have two functions that are related but independent. First, the beneficial innate immune activation improves Mtb control transiently but incompletely, then impaired lipid clearance induces ISR and cellular dysfunction later in the course of infection. There is precedent for a dual role for TOLLIP, as it balances clearance of insoluble endoplasmic reticulum proteins with stabilizing the cytoplasmic DNA sensor STING ${ }^{15}$. These findings may provide an explanation for the biphasic response to Mtb seen in Tollip ${ }^{-/-}$mice.

AM are the primary cell infected by Mtb during early infection, but their role during chronic infection is less well understood ${ }^{34,58}$. Chronically activated AM disproportionately contribute to immunopathology in influenza and RSV infection ${ }^{32,59}$ and exacerbate fibrotic responses in autoimmune diseases and idiopathic pulmonary fibrosis ${ }^{60}$. Our data suggest that TOLLIP prevents AM dysregulation during Mtb infection, and its absence leads to Mtb-induced immunopathology. These data identify a novel, genetically regulated role for AM during chronic Mtb disease. Specifically targeting therapeutics to AM during chronic Mtb infection may be effective for controlling persisting Mtb bacilli.

Tollip ${ }^{-/-}$mice demonstrated increased EIF2 phosphorylation in AM and in vivo, leading to immune pathology and Mtb progression. In prior studies, EIF2 was induced in Mtb-infected bone marrow-derived macrophages from sst1.B6 mice that produce excess Type I interferon ${ }^{47}$. Tollip ${ }^{-/-}$cells demonstrated impaired Type I interferon secretion compared to B6 mice due to decreased STING stability ${ }^{15}$, suggesting an alternate mechanism for ISR activation. MA induced the ISR in Mtb-infected, Tollip ${ }^{-/-}$macrophages ${ }^{48}$. In models of hepatic steatosis and atherosclerosis, lipid accumulation in the ER induces EIF2 phosphorylation ${ }^{61,62,63}$. Lipid-induced EIF2 activation may interact with inflammatory stress. Excess TNF induces programmed necrosis and Mtb spread by sphingosine-induced calcium flux and oxidative stress $^{24,25,64}$. Notably, we found strong enrichment of lipid and sphingolipid metabolic transcriptional pathways in Mtb-infected Tollip $^{-/-}$AM, and MA metabolizes into sphingosine in macrophages ${ }^{65}$. In summary, TOLLIP contributes to the degradation of Mtb-derived lipids to protect from Mtb immunopathology.

EIF2 activation and the downstream ISR may play an important role in the transition from asymptomatic Mtb infection to symptomatic TB disease. Studies from the preantibiotic era regularly describe "postprimary" tuberculosis as an acute, paucibacillary, caseating lipoid pneumonia obstructing bronchioles in a "tree-in-bud" pattern ${ }^{66,67}$. Within the lipoid pneumonia, obstructed alveoli attract macrophages with a foamy appearance, leading to subsequent necrosis and granuloma formation. Pathologically, this obstructive lipid pneumonia precedes the transition from asymptomatic to symptomatic TB disease ${ }^{39}$. Our model mimics important aspects of this observation, whereby Tollip ${ }^{-/-}$AM accumulate lipid, which permits Mtb growth via the EIF2 pathway. A combination of host and bacterial factors that that obstruct clearance of lipid debris may contribute to Mtb progression by inducing EIF2 in AM. 
This study revealed a critical role for TOLLIP within AM that governs immunopathology and Mtb infection outcomes. However, TOLLIP is ubiquitously expressed and may influence the function of other immune cell subsets relevant for Mtb host responses, particularly $T$ cells and dendritic cells. EIF2 signaling influences dendritic cell function, particularly in tumors and during chronic viral infections ${ }^{68,69,70}$. TOLLIP may also impact $T$ cell activation and differentiation. The selective autophagy receptor TAX1BP1, which is structurally and functionally similar to TOLLIP, is essential for the biosynthetic demands of T cells during the metabolically and synthetically demanding initial proliferative period ${ }^{71}$. Thus, future studies are needed to investigate how TOLLIP and ISR signaling pathways shape immune function across tissues and immune lineages.

We showed that ISRIB treatment improved Mtb control even after infection was established, a critical requirement for translation of any host-directed therapy to human disease. Often host-directed interventions are tested in mice by administration either prior to or very early after infection and may show lesser efficacy when used during chronic infection ${ }^{72,73,74}$. However, our studies show that the EIF2 pathway is required to clear lipids that would otherwise impair immunity during prolonged infection, providing a promising host-directed target for established disease. Importantly, we showed that EIF2inhibition enhanced immunity in both TOLLIP-deficient and B6 hosts. Thus, ISRIB is candidate hostdirected therapeutic agent that should be explored further in future studies.

\section{Online Methods}

\section{Reagents}

A complete list of reagents can be found in Table S1.

\section{Mice}

All mice were housed and maintained in specific pathogen-free conditions at the University of Washington and Seattle Children's Research Institute, and all experiments were performed in compliance with the Institutional Animal Care and Use Committee from each institution. Mice used in the experiments

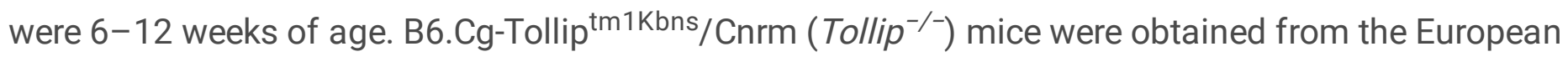
Mutant Mouse Archive (www.infrafrontier.eu) ${ }^{75}$. Mice were backcrossed 11 times on C57BL/6J background and were confirmed to be $>99 \%$ C57BL/6J genetically by screening 150 SNP ancestry informative markers (Jax, Inc). Genotyping was performed using DNA primers for neomycin (Forward sequence: AGG ATC TCC TGT CAT CTC ACC TTG CTC CTG; Reverse sequence AAG AAC TCG TCA AGA AGG CGA TAG AAG GCG) and the first exon of TOLLIP (Forward sequence: AGC TAC TGG GAG GCC ATA CA; Reverse sequence: CGT GTA CGG GAG ACC CAT TT). Protein expression was confirmed in both knockout and backcrossed alleles by qPCR and Western blot. All wild type control mice were age-matched littermates to ensure a common genetic background.

\section{Model of Mtb aerosol infection}


Aerosol infections were performed with Mtb, H37Rv strain transfected with a mCherry reporter plasmid, described previously ${ }^{34}$. Mice were enclosed in a Glas-Col aerosol infection chamber and $\sim 50-100 \mathrm{CFU}$ were deposited into mouse lungs. Doses were confirmed using control mice by plating lung homogenates on 7H10 agar immediately after aerosol infection. Mice were sacrificed at indicated timepoint, and lungs were gently homogenized in PBS-containing $0.05 \%$ Tween using a gentleMacs dissociator (Miltenyi Biotec). Tissue homogenates were serially diluted on 7H10 agar and lung CFU was enumerated.

\section{Lung Cell Flow Cytometry}

Lung single cell suspensions were washed and stained for viability with Zombie Aqua viability dye (BioLegend) for $10 \mathrm{~min}$ at room temperature in the dark. After incubation, $100 \mu \mathrm{l}$ of a surface antibody cocktail diluted in 50\% FACS buffer/50\% 24G2 Fc block buffer was added and surface staining performed for $30 \mathrm{~min}$ at $4^{\circ} \mathrm{C}$. Antibody lists can be found in Table S1. The cells were washed once with FACS buffer and fixed with $2 \%$ paraformaldehyde for $1 \mathrm{~h}$ prior to analyzing on an LSRII flow cytometer (BD Biosciences). In some experiments, intracellular staining was performed after surface staining. Permeabilization was performed with Fix-Perm buffer (eBiosciences) for minimum of 60 min before the addition of intracellular antibodies. Then cells were fixed with $2 \%$ paraformaldehyde for $1 \mathrm{~h}$ prior to analyzing on an LSRII flow cytometer (BD Biosciences). 28 days after infection, mice were sacrificed, and AM were sorted on a BD FACSAria in a BSL-3 facility for infected and uninfected populations. The samples were spun and stored $-80^{\circ} \mathrm{C}$ in Trizol.

\section{Chimera Generation}

:Tollip ${ }^{-/-}$mixed bone marrow chimeras were generated in the following manner: B6.SJL-Ptprca Pepcb/BoyJ (CD45.1+; Jax, Inc.) F1 mice were lethally irradiated (1000 cGy). 5-10x106 bone marrow cells comprised of a 1:1 mixture of CD3-depleted (Miltenyi Biotec) Tollip ${ }^{-/-}$(CD45.2+) and F1 generation of C57BL/6J (CD45.1 + 45.2+) bone marrow was provided intravenously. For full bone marrow chimera generation, and Tollip $^{-/-}$mice were irradiated, followed by hemopoietic reconstitution by adoptive transfer of $5-10 \times 10^{6}$ bone marrow cells via intravenous injection.

\section{Tissue Preparation and Evaluation}

Mice were euthanized and lungs were gently homogenized in HEPES buffer containing Liberase Blendzyme 3 (70 $\mu \mathrm{g} / \mathrm{ml}$; Roche) and DNasel ( $30 \mu \mathrm{g} / \mathrm{ml}$; Sigma-Aldrich) using a gentleMacs dissociator (Miltenyi Biotec). The lungs were then incubated for $30 \mathrm{~min}$ at $37^{\circ} \mathrm{C}$ and then further homogenized a second time with the gentleMacs. The homogenates were filtered through a $70 \mu \mathrm{m}$ cell strainer, pelleted for RBC lysis with RBC lysing buffer (Thermo), and resuspended in FACS buffer (PBS containing 2.5\% FBS and $0.1 \% \mathrm{NaN}_{3}$ ). To prepare organs for histology, lung sections were inflated to $15 \mathrm{~cm}$ water pressure with $4 \%$ paraformaldehyde, fixed in the same solution, embedded in paraffin, and sliced into $4 \mu \mathrm{m}$ sections. Sections stained with hematoxylin and eosin were examined by a pathologist blinded to mouse genotype. 
Frozen Mtb was thawed and cultured on a shaking incubator for two doubling cycles. One day prior to infection, cultures were back-diluted into an optical density (OD) of 0.2-0.4 in 7H9 media supplemented with glycerol (4\%), Middlebrook ADC Growth Supplement (100 mL/L), and Tween 80 (0.05\%). At the time of infection, Mtb was filtered through a $5 \mu \mathrm{m}$ syringe filter to remove bacterial clumps, and cells were inoculated at indicated MOI. The inoculum was prepared in RPMI-10 medium and applied to cells, which were centrifuged at $1200 \mathrm{rpm}$ for 5 minutes and incubated for 4 hours at $37^{\circ} \mathrm{C}$. Supernatants were removed and washed twice with prewarmed PBS to remove unbound bacteria, before adding per warmed RPMI supplemented with $10 \%$ FBS. Intracellular growth was determined using luminescence on a Synergy $\mathrm{H} 4$ multimode microplate reader (Biotek Instruments) daily from Day 0 to Day 3. In some experiments, $24 \mathrm{hrs}$ post infection cell culture supernatants were collected and filtered twice using $0.22 \mu \mathrm{m}$ filter and frozen at $-80^{\circ} \mathrm{C}$.

\section{Macrophage Preparation}

Resident peritoneal cells, mainly consisting of in vivo differentiated macrophages, were isolated using standard methods ${ }^{76}$. Briefly, $10 \mathrm{ml}$ of cold PBS was injected using $27 \mathrm{~g}$ needle, and peritoneum was gently messaged. Cells were removed with PBS, centrifuged at $4^{\circ} \mathrm{C}$ and placed into warm RPMI media. PEMs $\left(1 \times 10^{5}\right.$ /well) were plated in 96-well plates in $200 \mu \mathrm{L}$ of antibiotic-free RPMI 1640 containing with $10 \%$ Fetal Bovine Serum (Atlas Biologics). Cell were rested for minimum 24hrs before further experimentation. Bone marrow was harvested from mice and grown in RPMI supplemented with $10 \%$ heat inactivated FBS and M-CSF $(40 \mathrm{ng} / \mathrm{ml})$ in tissue culture treated plates. Cells were then incubated at $37^{\circ} \mathrm{C}$ for 6 to 7 days. Bone marrow-derived macrophages (BMDM) were used after 7 days of culture. BMDMs were detached by gentle scraping and were then plated in RPMI 1640 supplemented with 10\% heat inactivated FBS.

\section{Mycolic acid preparation}

a-mycolic acid (C80) was obtained from Avanti Polar Lipids. Powdered lipids were solubilized in chloroform, which was evaporated in the plate and the lipids recovered in RPMI with $10 \%$ FCS. The suspension was sonicated at $37^{\circ} \mathrm{C}$ for $20 \mathrm{~min}$, followed by addition of cells. Control suspensions were prepared similarly but without the addition of MA and sonicated before administration. $50 \mu \mathrm{g}$ of mycolic acid were administered to cells of interest for 72 hours prior to measurement, as previously described ${ }^{27}$. MA was free from endotoxin as determined by a chromogenic Limulus amebocyte lysate assay (Genscript, Piscatawy, NJ, USA).

\section{Cellular Studies}

Cell-culture supernatants were collected at indicated time and frozen at $-20^{\circ} \mathrm{C}$ until analysis. The cytokine concentrations in the culture supernatant were determined using quantitative ELISA (Mouse TNF and IL10 DuoSet; R\&D Systems) as recommended by the manufacturer. For detection of lipid bodies, PEMs from B6 and Tollip ${ }^{-/-}$mice were plated $\left(1 \times 10^{5} /\right.$ well) in a glass bottom chamber slide. Cells were washed twice and resuspended in PBS solution of HCS LipidTOX Deep Red Neutral Lipid stain (ThermoFisher Scientific) according to manufacturer instruction. After incubation, cells were washed twice with PBS and 
fixed in $2 \%$ PFA for $30 \mathrm{~min}$. Cells were mounted in medium containing DAPI stain (ProLong Gold, Thermo, Inc.). Lipid droplets were counted from 100 cells identified randomly selected high-powered fields.

\section{Western blotting}

Immunoblots were performed as described previously ${ }^{77}$. Briefly, 5-20 $\mu \mathrm{g}$ of cell or tissue protein extract was separated by SDS-PAGE, transferred onto PVDF membranes and immunoblotted with primary antibodies, listed in Table S1. Secondary antibodies conjugated to horseradish peroxidase were added and luminescence was quantitated.

\section{Preparation of total RNA and sequencing}

Total RNA from sorted cells was isolated using the manufacturer's instructions (TRIzol, Invitrogen). The RNA purity and quantified was assessed by RNA-Tapestation (Agilent 4200). cDNA was prepared using SMART-Seq v4 Ultra Low Input RNA Kit (Takara Bio USA, Inc). RNA sequencing libraries were prepared using the Illumina TruSeq ${ }^{\text {TM }}$ RNA Sample Preparation Kit (Illumina, San Diego, CA, USA). Samples were sequenced as 50 bp paired-end reads on a Illumina HiSeq 2500 and assessed using FastQC v0.11.8 to visualize sequence quality ${ }^{78}$. Sequences were filtered using AdapterRemoval v2.3.1 to remove adapters, remove sequences with $>1$ ambiguous base, and trim ends to a $30+$ Phred score ${ }^{79}$. Sequences were aligned to the mouse genome mm10 using STAR v2.7.4a ${ }^{80}$, and alignments were assessed with Picard v2.18.7 81 and samtools v1.10 ${ }^{82}$. Total reads in gene exons were quantified using featureCounts v2.0.1 83

\section{Differential expression analysis}

Gene expression analyses were performed in R v3.6.1 with the tidyverse v1.3.0 ${ }^{84}$. Overall, samples were very high-quality with > 32 million aligned sequences per sample and low variability in gene coverage (median coefficient of variation coverage $<0.6$ ). Genes were filtered to protein coding genes with biomaRt $(N=21850)$ with $>0.1$ counts per million $(C P M)$ in at least three samples $(N=14215){ }^{85}$. Counts were normalized for RNA composition using edgeR and log2 CPM voom normalized with quality weights using limma 86,87 . Differentially expressed genes were determined using limma using a contrasts model comparing B6 and Tollip ${ }^{-/-}$cells in uninfected and infected groups (Table S2). Genes with FDR $<0.3$ for at least one contrast $(\mathrm{N}=3899)$ were included in Ingenuity Pathway analysis. The Benjamini-Hochberg correction for multiple comparisons was applied to P-values and significance was assessed at FDR $<0.05$ for all tests.

\section{Statistics}

For mouse experiments, groups of five mice were compared with one another unless otherwise indicated. Cellular measurements were compared using a two-sided $t$-test unless otherwise specified. A value of $p<$ 0.05 was considered a statistically significant result unless otherwise specified. Statistics were calculated using Prism version 8.0 (GraphPad, Inc.). 


\section{Declarations}

\section{Acknowledgments}

The authors wish to thank the University of Washington Center for Lung Biology Histology and Imaging Core for their helpful advice on pathology staining and analysis. We are grateful to the Seattle Children's Research Institute Cell Sorting Core for their assistance and technical support. We thank Lalita Ramakrishnan for helpful conversations. Funding: This work was supported by the NIH (R01 Al136912 to JAS; R01 DK108921 to SAS) and the Department of Veterans Affairs (I01 BX004444 to SAS). GLP was supported by the American Diabetes Association (19-PDF-063).

Author Contributions: Conceptualization: SV, KU, SS JS; methodology: SV, SS, KU, MA, SD, JS; resources: JS, software: KDM, MA; validation: SV, JS, RE; formal analysis: MA, KDM; investigation: SV, SH, CP, GP, AL, AP, RP, JS; writing - original draft: SV, JS; writing - review and editing: KU, SS, MA, CP, JS; supervision: JS, $\mathrm{KU}, \mathrm{MA}$, SS; project administration: SV, JS; funding acquisition: JS, KU, SS, MA.

Competing Interest Statement: The authors declare no competing interests

\section{References}

1. Shah, J.A. et al. Human TOLLIP Regulates TLR2 and TLR4 Signaling and Its Polymorphisms Are Associated with Susceptibility to Tuberculosis. J Immunol 189, 1737-1746 (2012).

2. Shah, J.A. et al. A Functional TOLLIP Variant is Associated with BCG-Specific Immune Responses and Tuberculosis. Am J Respir Crit Care Med (2017).

3. Shah, J.A. et al. Genetic Variation in Toll-Interacting Protein Is Associated With Leprosy Susceptibility and Cutaneous Expression of Interleukin 1 Receptor Antagonist. J Infect Dis 213, 1189-1197 (2016).

4. Shih, S.C. et al. A ubiquitin-binding motif required for intramolecular monoubiquitylation, the CUE domain. Embo J 22, 12731281 (2003).

5. Uhlén, M. et al. Proteomics. Tissue-based map of the human proteome. Science 347, 1260419 (2015).

6. Jongsma, M.L. et al. An ER-Associated Pathway Defines Endosomal Architecture for Controlled Cargo Transport. Cell 166, 152-166 (2016).

7. Chen, K., Yuan, R., Zhang, Y., Geng, S. \& Li, L. Tollip Deficiency Alters Atherosclerosis and Steatosis by Disrupting Lipophagy. J Am Heart Assoc 6, e004078 (2017).

8. Lu, K., Psakhye, I. \& Jentsch, S. Autophagic clearance of polyQ proteins mediated by ubiquitin-Atg8 adaptors of the conserved CUET protein family. Cell 158, 549-563 (2014).

9. Shah, J.A. et al. TOLLIP deficiency is associated with increased resistance to Legionella pneumophila pneumonia. Mucosal Immunol 12, 1382-1390 (2019).

10. Burns, K. et al. Tollip, a new component of the IL-1RI pathway, links IRAK to the IL-1 receptor. Nat Cell Biol 2, 346-351 (2000). 
11. Ryan, T.A. et al. Tollip coordinates Parkin-dependent trafficking of mitochondrial-derived vesicles. The EMBO Journal 39, e102539 (2020).

12. Zellner, S., Schifferer, M. \& Behrends, C. Systematically defining selective autophagy receptor-specific cargo using autophagosome content profiling. Mol Cell (2021).

13. Zhang, G., Ghosh, S. Negative Regulation of Toll-like Receptor-mediated Signaling by Tollip. Journal of Biological Chemistry 277, 7059-7065 (2002).

14. Brissoni, B. et al. Intracellular trafficking of interieukin-1 receptor I requires Tollip. Curr. Biol. 16, 22652270 (2006).

15. Pokatayev, V. et al. Homeostatic regulation of STING protein at the resting state by stabilizer TOLLIP. Nat Immunol 21, 158-167 (2020).

16. Kowalski, E.J.A. \& Li, L. Toll-Interacting Protein in Resolving and Non-Resolving Inflammation. Front Immunol 8, 511 (2017).

17. Noth, I. et al. Genetic variants associated with idiopathic pulmonary fibrosis susceptibility and mortality: a genome-wide association study. Lancet Respir Medicine 1, 309-317 (2013).

18. Li, M. et al. Tollip is a critical mediator of cerebral ischaemia-reperfusion injury. J Pathology 237, 249-262 (2015).

19. Zhi, H. et al. Tollip Negatively Regulates Vascular Smooth Muscle Cell-Mediated Neointima Formation by Suppressing Akt-Dependent Signaling. J Am Heart Assoc 7 (2018).

20. Feng, C.G. et al. Mice lacking myeloid differentiation factor 88 display profound defects in host resistance and immune responses to Mycobacterium avium infection not exhibited by Toll-like receptor 2 (TLR2)- and TLR4-deficient animals. J Immunol 171, 4758-4764 (2003).

21. Bafica, A. et al. TLR9 regulates Th1 responses and cooperates with TLR2 in mediating optimal resistance to Mycobacterium tuberculosis. Journal of Experimental Medicine 202, 1715-1724 (2005).

22. Watson, R.O. et al. The Cytosolic Sensor cGAS Detects Mycobacterium tuberculosis DNA to Induce Type I Interferons and Activate Autophagy. Cell Host Microbe 17, 811-819 (2015).

23. Philips, J.A., Porto, M.C., Wang, H., Rubin, E.J. \& Perrimon, N. ESCRT factors restrict mycobacterial growth. Proceedings of the National Academy of Sciences of the United States of America 105, 3070-3075 (2008).

24. Roca, F.J. \& Ramakrishnan, L. TNF dually mediates resistance and susceptibility to mycobacteria via mitochondrial reactive oxygen species. Cell 153, 521-534 (2013).

25. Roca, F.J., Whitworth, L.J., Redmond, S., Jones, A.A. \& Ramakrishnan, L. TNF Induces Pathogenic Programmed Macrophage Necrosis in Tuberculosis through a Mitochondrial-LysosomalEndoplasmic Reticulum Circuit. Cell 178, 1344-1361 e1311 (2019).

26. Lovewell, R.R., Sassetti, C.M. \& VanderVen, B.C. Chewing the fat: lipid metabolism and homeostasis during M. tuberculosis infection. Curr Opin Microbiol 29, 30-36 (2016). 
27. Peyron, P. et al. Foamy macrophages from tuberculous patients' granulomas constitute a nutrientrich reservoir for M. tuberculosis persistence. PLoS Pathog 4, e1000204 (2008).

28. Berg, R.D. et al. Lysosomal Disorders Drive Susceptibility to Tuberculosis by Compromising Macrophage Migration. Cell 165, 139-152 (2016).

29. Pagán, A.J. \& Ramakrishnan, L. Immunity and Immunopathology in the Tuberculous Granuloma. Cold Spring Harbor Perspectives in Medicine 5 (2015).

30. Consortium, G.T. Human genomics. The Genotype-Tissue Expression (GTEx) pilot analysis: multitissue gene regulation in humans. Science 348, 648-660 (2015).

31. Russell, D.G., Huang, L. \& VanderVen, B.C. Immunometabolism at the interface between macrophages and pathogens. Nat Rev Immunol 19, 291-304 (2019).

32. Snelgrove, R.J. et al. A critical function for CD200 in lung immune homeostasis and the severity of influenza infection. Nature immunology 9, 1074-1083 (2008).

33. Guilliams, M. et al. Alveolar macrophages develop from fetal monocytes that differentiate into longlived cells in the first week of life via GM-CSF. J Exp Med 210, 1977-1992 (2013).

34. Cohen, S.B. et al. Alveolar Macrophages Provide an Early Mycobacterium tuberculosis Niche and Initiate Dissemination. Cell Host Microbe 24, 439-446 e434 (2018).

35. Krämer, A., Green, J., Pollard, J., Jr. \& Tugendreich, S. Causal analysis approaches in Ingenuity Pathway Analysis. Bioinformatics 30, 523-530 (2014).

36. Hinnebusch, A.G. Mechanism and regulation of initiator methionyl-tRNA binding to ribosomes. COLD SPRING HARBOR MONOGRAPH SERIES 39, 185-244 (2000).

37. Crowe, A. \& Yue, W. Semi-quantitative Determination of Protein Expression Using Immunohistochemistry Staining and Analysis: An Integrated Protocol. Bio-protoco/ 9 (2019).

38. Hunter, R.L. On the pathogenesis of post primary tuberculosis: the role of bronchial obstruction in the pathogenesis of cavities. Tuberculosis (Edinb) 91 Suppl 1, S6-10 (2011).

39. Hunter, R.L. Tuberculosis as a three-act play: A new paradigm for the pathogenesis of pulmonary tuberculosis. Tuberculosis (Edinb) 97, 8-17 (2016).

40. Grootjans, J., Kaser, A., Kaufman, R.J. \& Blumberg, R.S. The unfolded protein response in immunity and inflammation. Nat Rev Immunol 16, 469-484 (2016).

41. Kim, M.J. et al. Caseation of human tuberculosis granulomas correlates with elevated host lipid metabolism. EMBO Mol Med 2, 258-274 (2010).

42. Berry, M.P.R. et al. An interferon-inducible neutrophil-driven blood transcriptional signature in human tuberculosis. Nature; 2010. pp. 973-977.

43. Uchida, K. et al. Activation of stress signaling pathways by the end product of lipid peroxidation: 4hydroxy-2-nonenal is a potential inducer of intracellular peroxide production. Journal of Biological Chemistry 274, 2234-2242 (1999).

44. Cubillos-Ruiz, J.R. et al. ER Stress Sensor XBP1 Controls Anti-tumor Immunity by Disrupting Dendritic Cell Homeostasis. Cell 161, 1527-1538 (2015). 
45. Manzo, T. et al. Accumulation of long-chain fatty acids in the tumor microenvironment drives dysfunction in intrapancreatic CD8 + T cells. J Exp Med 217 (2020).

46. Costa-Mattioli, M. \& Walter, P. The integrated stress response: From mechanism to disease. Science 368 (2020).

47. Bhattacharya, B. et al. The integrated stress response mediates necrosis in murine Mycobacterium tuberculosis granulomas. J Clin Invest (2020).

48. Vermeulen, I. et al. Mycolates of Mycobacterium tuberculosis modulate the flow of cholesterol for bacillary proliferation in murine macrophages. J Lipid Res 58, 709-718 (2017).

49. Ouimet, M. et al. Autophagy regulates cholesterol efflux from macrophage foam cells via lysosomal acid lipase. Cell Metab 13, 655-667 (2011).

50. Zechner, R., Madeo, F. \& Kratky, D. Cytosolic lipolysis and lipophagy: two sides of the same coin. Nature Reviews Molecular Cell Biology 18, 671-684 (2017).

51. Klionsky, D.J. et al. Guidelines for the use and interpretation of assays for monitoring autophagy (4th edition)1. Autophagy 17, 1-382 (2021).

52. Nicolaou, G. \& Erridge, C. Toll-like receptor-dependent lipid body formation in macrophage foam cell formation. Curr Opin Lipidol 21, 427-433 (2010).

53. Nataraj, V. et al. Mycolic acids and the tubercle bacillus. Mol Microbiol 98, 7-16 (2015).

54. Fu, S. et al. Aberrant lipid metabolism disrupts calcium homeostasis causing liver endoplasmic reticulum stress in obesity. Nature 473, 528-531 (2011).

55. Krzyzosiak, A. et al. Target-Based Discovery of an Inhibitor of the Regulatory Phosphatase PPP1R15B. Cell 174, 1216-1228 e1219 (2018).

56. Rabouw, H.H. et al. Small molecule ISRIB suppresses the integrated stress response within a defined window of activation. Proceedings of the National Academy of Sciences of the United States of America 116, 2097-2102 (2019).

57. Cardiff, R.D., Miller, C.H. \& Munn, R.J. Manual Hematoxylin and Eosin Staining of Mouse Tissue Sections. Cold Spring Harbor Protocols 2014, pdb.prot073411 (2014).

58. Rothchild, A.C. et al. Alveolar macrophages generate a noncanonical NRF2-driven transcriptional response to Mycobacterium tuberculosis in vivo. Sci Immunol 4 (2019).

59. Bem, R.A. et al. Potential role of soluble TRAIL in epithelial injury in children with severe RSV infection. American journal of respiratory cell and molecular biology 42, 697-705 (2010).

60. Gibbons, M.A. et al. Ly6Chi monocytes direct alternatively activated profibrotic macrophage regulation of lung fibrosis. American journal of respiratory and critical care medicine 184, 569-581 (2011).

61. Han, J. \& Kaufman, R.J. The role of ER stress in lipid metabolism and lipotoxicity. J Lipid Res 57, 1329-1338 (2016).

62. Cullinan, S.B. \& Diehl, J.A. Coordination of ER and oxidative stress signaling: The PERK/Nrf2 signaling pathway. The International Journal of Biochemistry \& Cell Biology 38, 317-332 (2006). 
63. Qin, S., Yin, J. \& Huang, K. Free Fatty Acids Increase Intracellular Lipid Accumulation and Oxidative Stress by Modulating PPARa and SREBP-1c in L-02 Cells. Lipids 51, 797-805 (2016).

64. Almaguel, F.G. et al. Lipotoxicity-mediated cell dysfunction and death involve lysosomal membrane permeabilization and cathepsin L activity. Brain Res 1318, 133-143 (2010).

65. Fineran, P. et al. Pathogenic mycobacteria achieve cellular persistence by inhibiting the Niemann-Pick Type C disease cellular pathway. Wellcome Open Res 1, 18 (2016).

66. Cornil, V. \& Ranvier, L. A manual of pathological histology translated with notes and additions by EO Shakespeare and JHC Simms. Philadephia: Henry C Lea, 394-445 (1880).

67. Im, J.G., Itoh, H., Lee, K.S. \& Han, M.C. CT-pathology correlation of pulmonary tuberculosis. Crit Rev Diagn Imaging 36, 227-285 (1995).

68. Mendes, A. et al. Proteostasis in dendritic cells is controlled by the PERK signaling axis independently of ATF4. Life Sci Alliance 4 (2021).

69. Ravindran, R. et al. Vaccine activation of the nutrient sensor GCN2 in dendritic cells enhances antigen presentation. Science 343, 313-317 (2014).

70. Poropatich, K. et al. OX40 + plasmacytoid dendritic cells in the tumor microenvironment promote antitumor immunity. J Clin Invest 130, 3528-3542 (2020).

71. Whang, M.I. et al. The Ubiquitin Binding Protein TAX1BP1 Mediates Autophagasome Induction and the Metabolic Transition of Activated T Cells. Immunity 46, 405-420 (2017).

72. Hawn, T.R., Shah, J.A. \& Kalman, D. New tricks for old dogs: countering antibiotic resistance in tuberculosis with host-directed therapeutics. Immunol Rev 264, 344-362 (2015).

73. Schiebler, M. et al. Functional drug screening reveals anticonvulsants as enhancers of mTORindependent autophagic killing of Mycobacterium tuberculosis through inositol depletion. EMBO Molecular Medicine 7, 127-139 (2015).

74. Napier, R.J. et al. Imatinib-sensitive tyrosine kinases regulate mycobacterial pathogenesis and represent therapeutic targets against tuberculosis. Cell Host Microbe 10, 475-485 (2011).

75. Didierlaurent, A. et al. Tollip regulates proinflammatory responses to interleukin-1 and lipopolysaccharide. Mol Cell Biol 26, 735-742 (2006).

76. Zhang, X., Goncalves, R. \& Mosser, D.M. The isolation and characterization of murine macrophages. Curr Protoc Immunol Chap. 14, Unit 1411 (2008).

77. Soleimanpour, S.A. et al. The diabetes susceptibility gene Clec16a regulates mitophagy. Cell 157 , 1577-1590 (2014).

78. Andrews, S. FastQC: A Quality Control Tool for High Throughput Sequence Data. 2010.

79. Schubert, M., Lindgreen, S. \& Orlando, L. AdapterRemoval v2: rapid adapter trimming, identification, and read merging. BMC research notes 9, 88-88 (2016).

80. Dobin, A. et al. STAR: ultrafast universal RNA-seq aligner. Bioinformatics (Oxford, England) 29, 1521 (2013).

81. Picard Toolkit. Broad Institute; 2019. 
82. Li, H. et al. The Sequence Alignment/Map format and SAMtools. Bioinformatics (Oxford, England) 25, 2078-2079 (2009).

83. Liao, Y., Smyth, G.K. \& Shi, W. featureCounts: an efficient general purpose program for assigning sequence reads to genomic features. Bioinformatics 30, 923-930 (2013).

84. Wickham, H. et al. Welcome to the Tidyverse. Journal of Open Source Software 4, 1686-1686 (2019).

85. Durinck, S. et al. BioMart and Bioconductor: a powerful link between biological databases and microarray data analysis. Bioinformatics 21, 3439-3440 (2005).

86. Robinson, M.D., McCarthy, D.J. \& Smyth, G.K. edgeR: a Bioconductor package for differential expression analysis of digital gene expression data. Bioinformatics (Oxford, England) 26, 139-140 (2010).

87. Ritchie, M.E. et al. limma powers differential expression analyses for RNA-sequencing and microarray studies. Nucleic acids research 43, e47-e47 (2015).

\section{Figures}




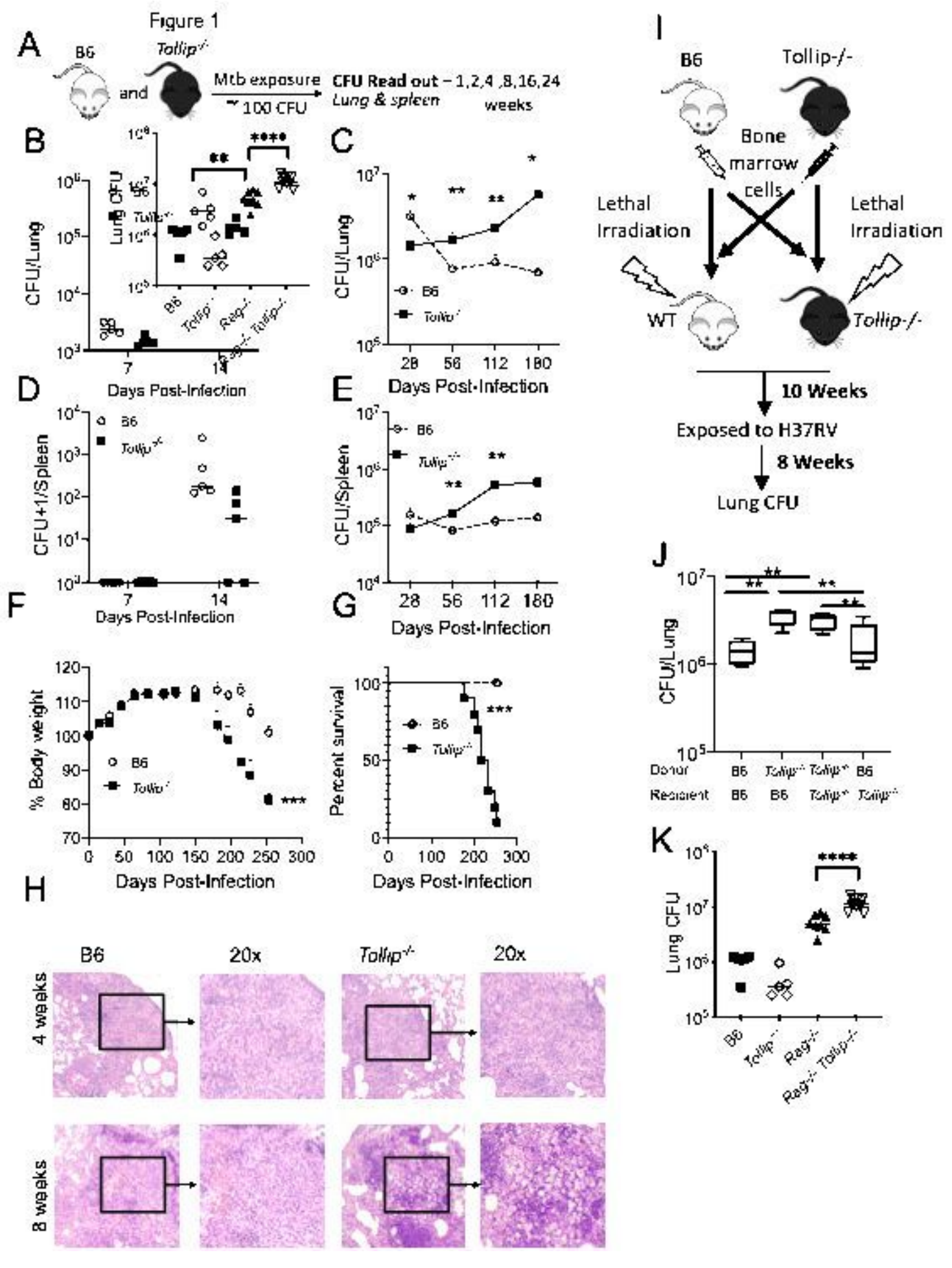

Figure 1

TOLLIP is required for Mtb control in mice and its absence induces foam cell formation. Mice were infected with Mtb H37Rv (50-100 CFU) via aerosol and monitored over time.
A) Experimental timeline.

B-C) Lung bacterial burden in B6 (clear circle) and Tollip ${ }^{-1-}$ (black square) mice B) 1 and2 weeks after infection and C) 4 weeks and onward. ${ }^{*} p<0.05$, ${ }^{* *} p<0.01{ }^{* * *} p<0.001$ at each time point; two-sided t- 
test at each time point; $n=50$. Overall association between genotypes $-p=0.0123$; mixed effects model.

D-E) Spleen bacterial burden D) 1 and 2 weeks after infection and E) 4 weeks and later after infection. * $p$ $<0.05$; $* \star p<0.01$, ${ }^{\star \star \star} p<0.001$, two-sided t-test at each time point; $\mathrm{n}=30$. Overall association between genotypes $-p=0.0092$; mixed effects model.

F) Percentage of initial body weight after Mtb infection Circle - B6 mice; square- Tollip ${ }^{-/}$mice. $\mathrm{N}=20$ *** $p<0.001$, two-way ANOVA accounting for time and genotype.

G) Survival curve analysis of B6 (clear circle) and Tollip ${ }^{-1}$ (black square) mice after aerosol infection with 50-100 CFU Mtb H37Rv. N = 20. *** $p<0.0001$, Mantel-Cox test.

H) Hematoxylin and eosin staining of Mtb-infected lung tissue from B6 and Tollip ${ }^{-/-}$mice 28 and 56 days after aerosol infection.

I) Experimental outline for bone marrow chimera experiments.

J) Lung bacterial burden of bone marrow chimeric mice, 56 days after aerosol Mtb infection. $N=5$ mice/group.

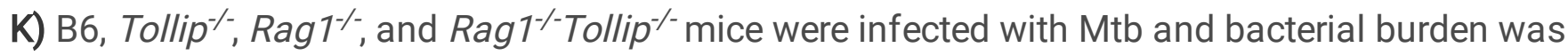
measured 28 days after infection ( $n=5-10$ mice/group). ${ }^{*} p<0.05$, $* * p<0.01, * * * p<0.001$, two-sided ttest. 
Frgure 2
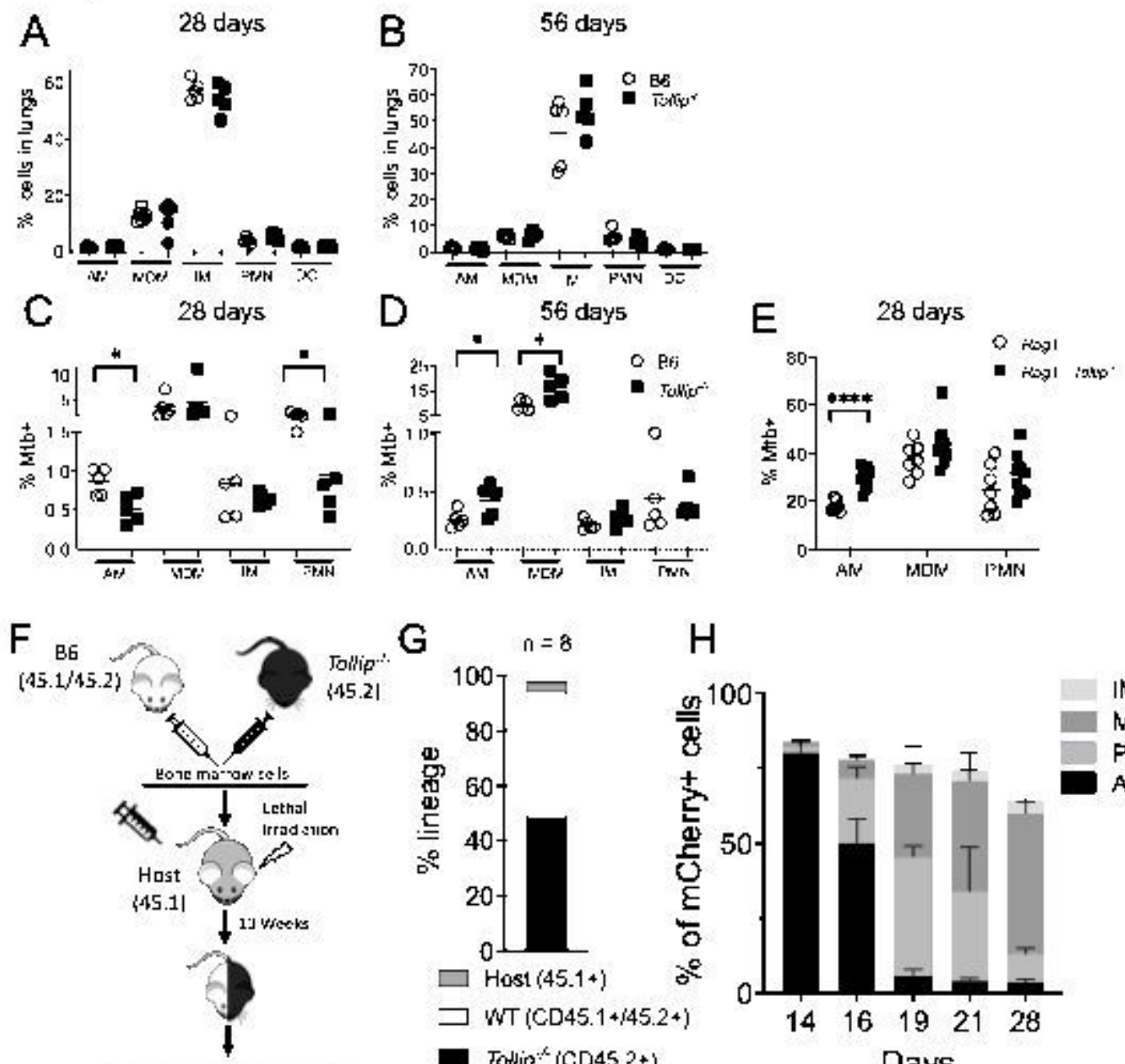

Exposed to H37Ru micherry

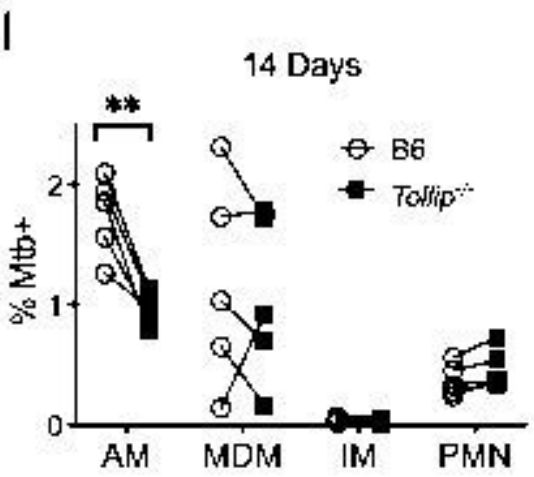

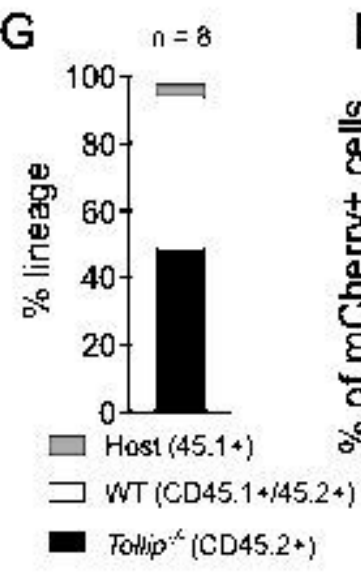

$J$

$\mathrm{H}$
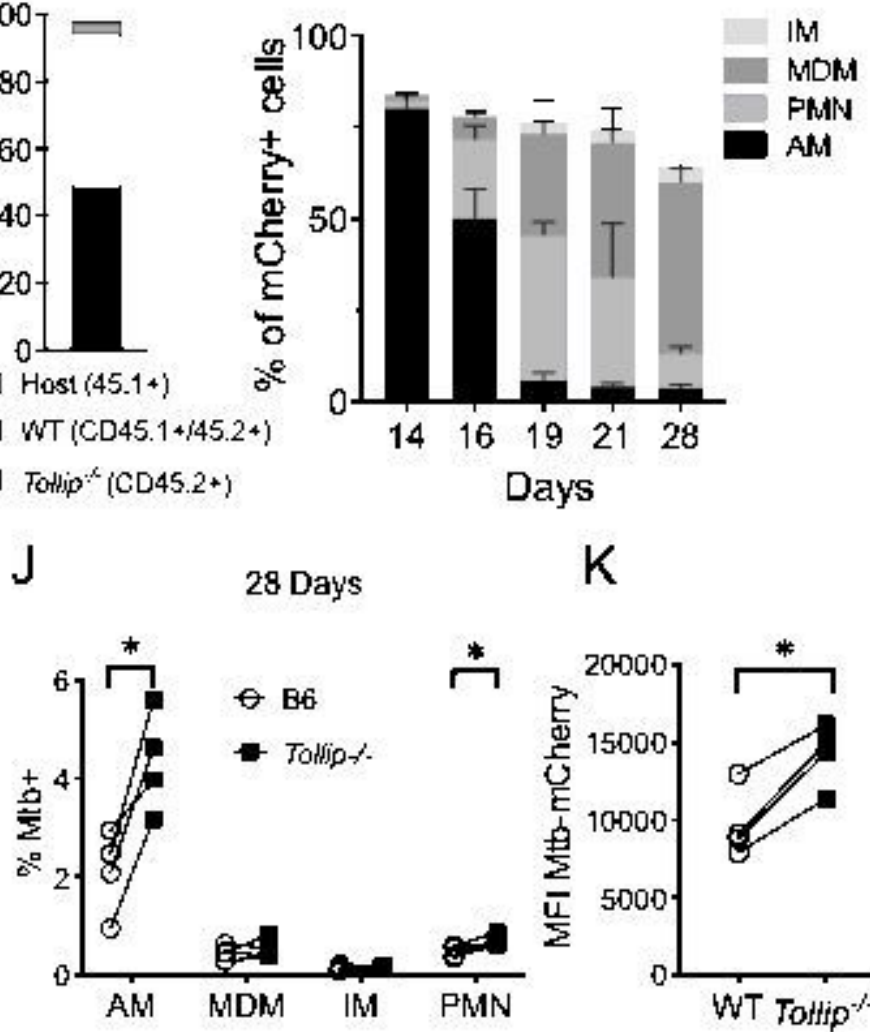

28 Days
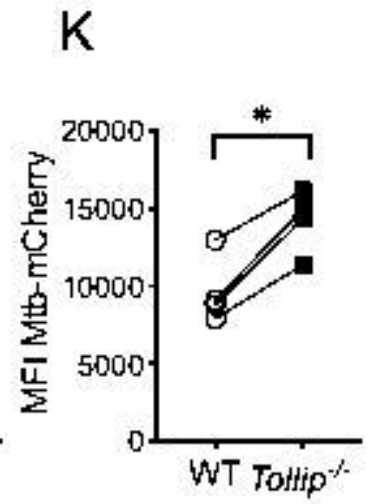

Figure 2

Tollip ${ }^{-1}$ AM are preferentially infected with Mtb due to intrinsic activity.

A-B) Lungs from Mtb-infected full knockout mice were gently dissociated, and flow cytometry was performed. Gating strategy is described in Figure S2. The proportion of AM, MDM, IM, and PMN from B6 (clear circle) and Tollip ${ }^{-/}$(black square) mice were compared A) 4 and B) 8 weeks after aerosol infection with 50-100 CFU mCherry-expressing Mtb; $n=5 ;$ * $p<0.05$, two-sided t-test. 
C-D) The proportion of Mtb-infected (mCherry+) AM, MDM, IM, and PMN rom B6 (clear circle) and Tollip ${ }^{-1-}$ (black square) mice were compared C) 28 and D) 56 days after infection. $n=5 /$ group; ${ }^{*} p<0.05$, twosided t-test.

E) The proportion of Mtb-infected AM, MDM, and PMN from Rag ${ }^{\%}$ (clear circle) and Rag ${ }^{\%-}$ Tollip ${ }^{-/}$mice

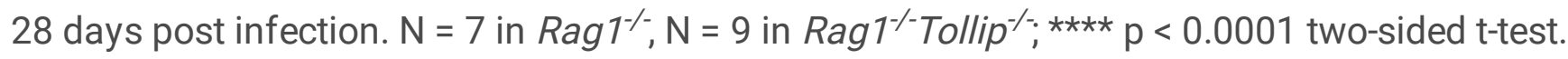

F) Mixed bone marrow chimera experimental strategy and timeline. Mixed bone marrow chimeras were generated by transferring a 1:1 mix of B6 (CD45.1+/CD45.2+) and Tollip ${ }^{-/}(\mathrm{CD} 45.2+)$ bone marrow to a CD45.1+ murine host. Immune cell reconstitution was permitted for 10 weeks. The gating strategy is described in Figure $\mathbf{S 4}$.

G) Distribution of CD45 expression in naïve mixed bone marrow chimeric mice. $\mathrm{N}=8$.

H) Composition of Mtb-infected lung myeloid cell types at multiple time points after aerosol infection with Mtb.

I-J) Distribution of Mtb-infected (mCherry+) lung-specific myeloid cells at $\mathrm{H}$ ) 14 and I) 28 days postinfection in mixed bone marrow chimeric mice stratified by B6 (white circle) and Tollip ${ }^{-1-}$ (black square) genotype. Lines connect genotypes from paired samples. ${ }^{*} p<0.05,{ }^{* \star} p<0.01$, paired two-sided t-test. $\mathrm{N}$ $=5 /$ group.

K) Median fluorescence intensity of mCherry in Mtb-infected (mCherry+) AM 8 days post-infection in mixed bone marrow chimeric mice stratified by B6 (white circle) and Tollip ${ }^{-/-}$(black square) genotype. Paired samples are connected by lines. ${ }^{*} \mathrm{p}<0.05$, paired two-sided t-test $\mathrm{N}=5$.

AM - alveolar macrophage, MDM - monocyte-derived macrophage, IM - interstitial macrophage, PMN neutrophil, DC - dendritic cell. Experiments were performed at least three times for reproducibility. 
Figure 3
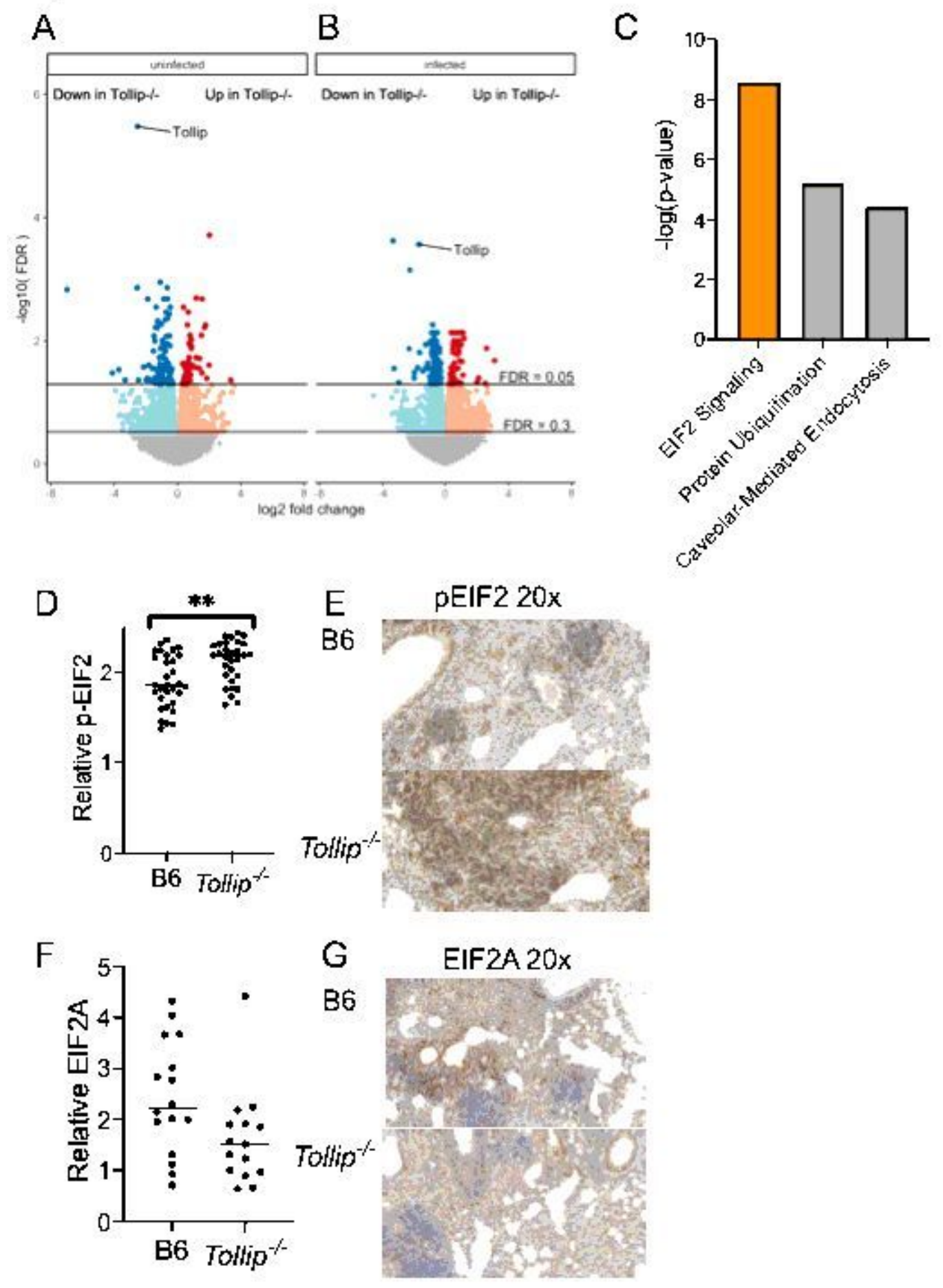

Figure 3

Tollip ${ }^{-1}$ AM develop increased EIF2 signaling after prolonged Mtb infection. Mixed bone marrow chimeric mice were infected with mCherry-expressing Mtb. 28 days after infection, Mtb-infected and Mtbuninfected B6 and Tollip ${ }^{-/}$AM were sorted and RNA-seq was performed. Sorting strategy can be found in Figure S3. 
A-B) Volcano plot of gene expression (log2 fold change) and significance (-log10(FDR)) for genes between Tollip ${ }^{-1}$ (CD45.2) and B6 (CD45.1+CD45.2+) in A) Mtb-uninfected and B) Mtb-infected AMs. Horizontal lines indicate significant genes (FDR $<0.05)$ and genes of interest $(F D R<0.3)$.

C) Ingenuity Causal Network Analysis on differentially expressed genes between B6 and Tollip ${ }^{-/}$Mtbinfected AM subgroup (FDR q < 0.3). P value cutoff $<10^{-4}$.

D) Semiquantitative analysis of EIF2 phosphorylated at Ser51 (pEIF2) expression in the lungs of B6 and Tollip ${ }^{-/}$mice eight weeks after Mtb infection.

E) Representative 20x imaging sections of lung tissue sections stained for pEIF2 by immunohistochemistry.

F) Semiquantitative analysis of EIF2S1 expression in the lungs of B6 and Tollip ${ }^{-/}$mice two months after Mtb infection.

G) Representative 20x imaging sections of lung tissue sections stained for pEIF2 by immunohistochemistry. ${ }^{*} p<0.05,{ }^{*} p<0.01$, two-sided t-test. $\mathrm{N}=5$ mice/group. 
Figure 4
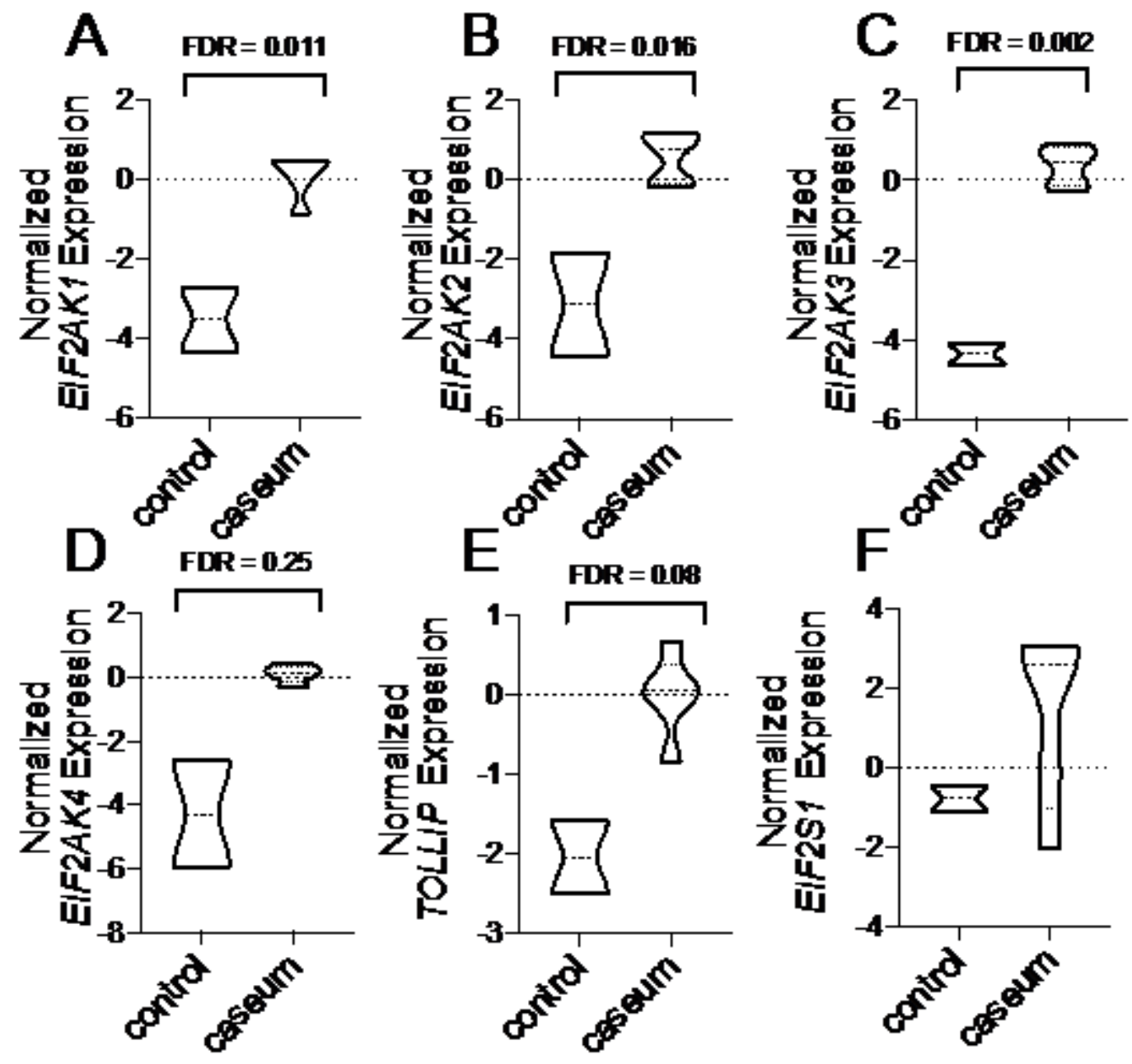

Figure 4

TOLLIP and EIF2 kinase transcripts are enriched in caseous TB granulomas.

A-F) Gene transcript expression of A) EIF2AK1, B) EIF2AK2, C) EIF2AK3, D) EIF2AK4, E) EIF2S1, and F) TOLLIP in human caseous granuloma tissue compared with healthy-appearing lung tissue from different lobes in the same individuals. Data are derived from the analysis of the publicly available GEO dataset GSE20050 using the indicated probe sets. FDR - false discovery rate. FDR is indicated above each data point. 
Figure 5

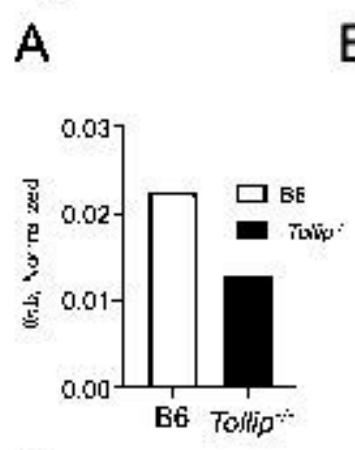

$E$

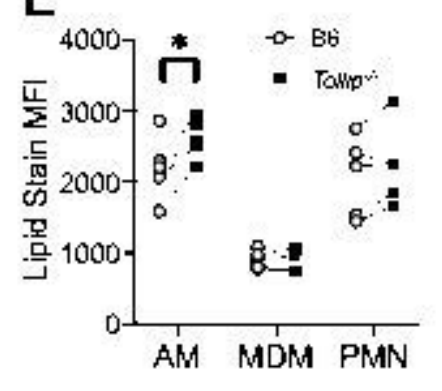

B $B 6$
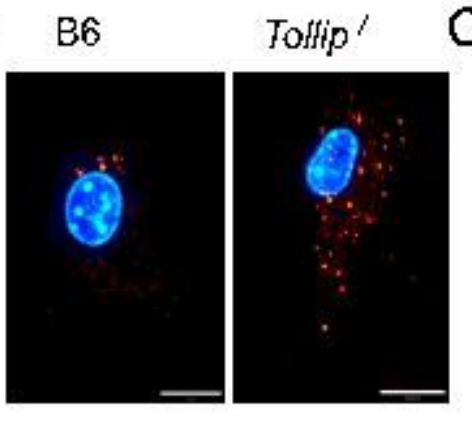

$\mathrm{F}$
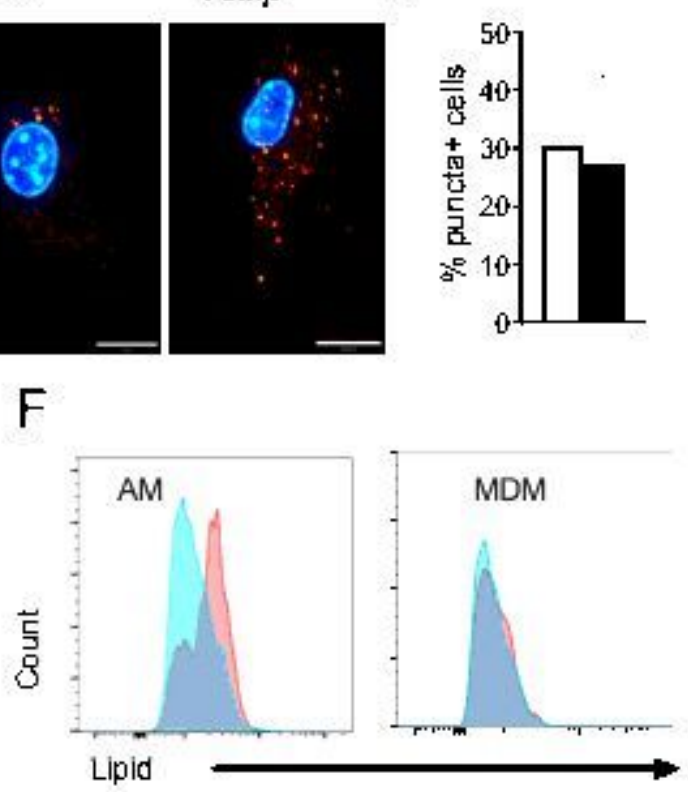
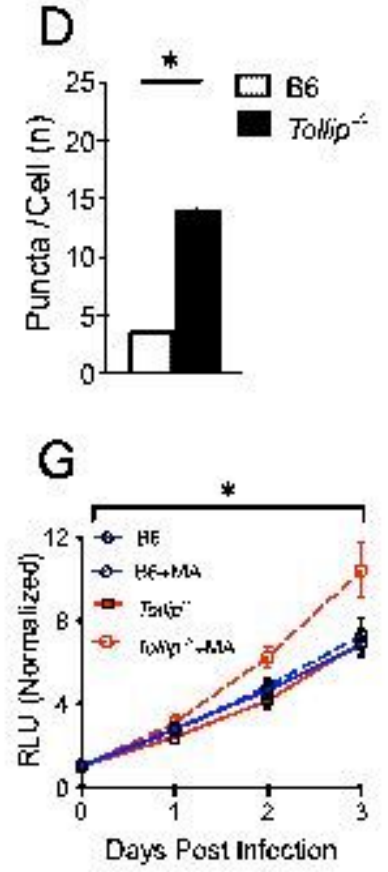

Figure 5

Tollip ${ }^{-/}$macrophages accumulate excess lipid and increased Mtb replication after mycolic acid incubation.

A-C) B6 and Tollip $\%$ peritoneal exudate macrophages (PEM) were isolated and incubated with Mtb cell wall lipid mycolic acid (MA) for 72hrs. PEM were stained with LipidTOX Red dye (red) and DAPI (b/ue), fixed, and visualized with fluorescent microscopy.
A) Representative image of lipid droplet (LD) accumulation in B6 and Tollip ${ }^{-/}$PEM.

B) Percentage of cells with visible LD. At least 100 cells imaged per experiment. Bar represents mean \pm SD values.

C) Total number of LD per cell of 100 cells with detectable LD. Bar - error bars (mean \pm SD).

D) Median fluorescence intensity of LipidTox neutral lipid stain from mixed bone marrow chimeric mice in alveolar macrophages (AM) and monocyte-derived macrophages (MDM) 28 days after Mtb infection, stratified by B6 (white circle) and Tollip ${ }^{-/}$(black square) genotype. Infection and gating were performed as in Figure S4. Lines connect B6 and Tollip ${ }^{-1-}$ AM from the same mouse. ${ }^{*} p<0.05$, paired two-sided ttest; $\mathrm{N}=5$. Experiment was performed five times.

E) Representative histogram of AM and MDM lipid staining (B6 - blue; Tollip ${ }^{-/-}$- red).

F) Luminescence of $\mathrm{B} 6$ and Tollip ${ }^{-/}$PEM incubated with mycolic acid (MA; $\left.10 \mu \mathrm{g} / \mathrm{ml}\right)$ and infected with Mtb H37Rv expressing luxCDABE luminescence gene from Vibrio harleyi (MOI 1). PEMs were isolated and 
incubated with MA for $72 \mathrm{hr}$ before infection. Luminescence was measured daily over 48-72 hours; $\mathrm{N}=6$. Experiments were repeated three times; all data is shown. * $p<0.05$; 3 -way ANOVA for an effect of MA in Tollip ${ }^{--}$PEM when controlling for time.

Figure 6

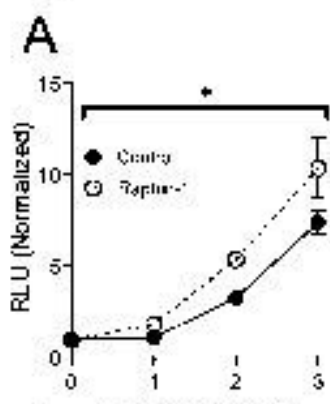

C Days Post Infection
B

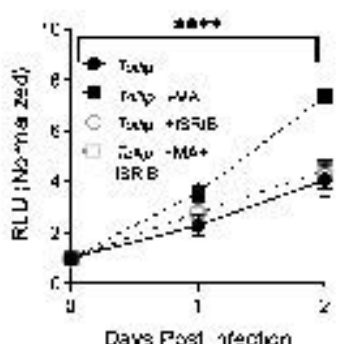

Days Fos Trecisn

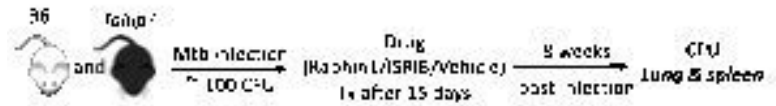

raphin-1

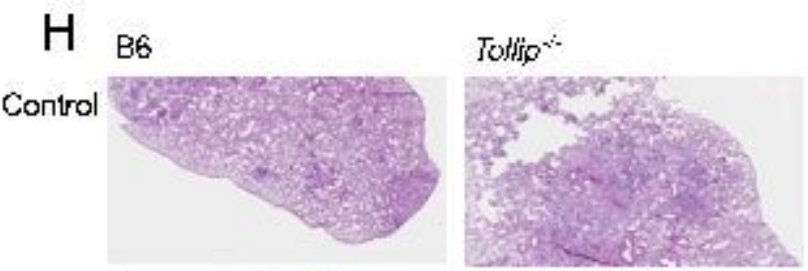

ISRIB
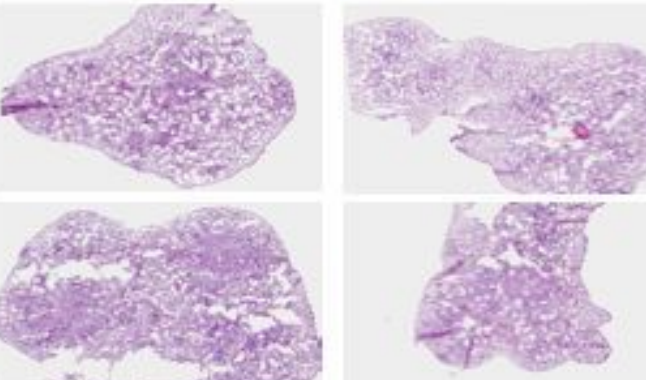

D
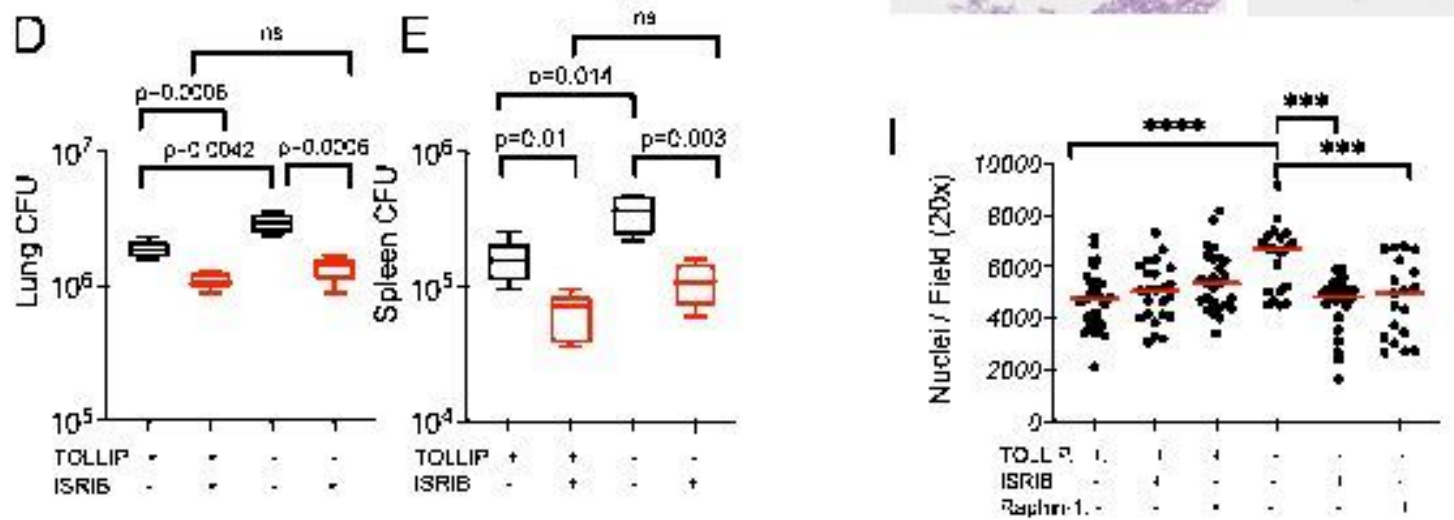

F

\section{G}

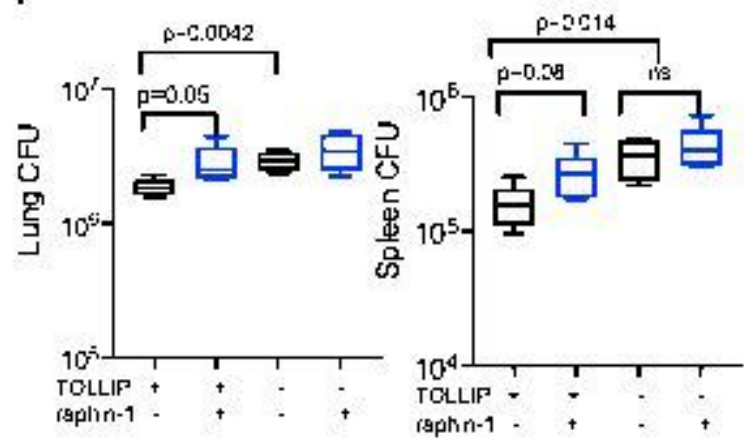

$J$
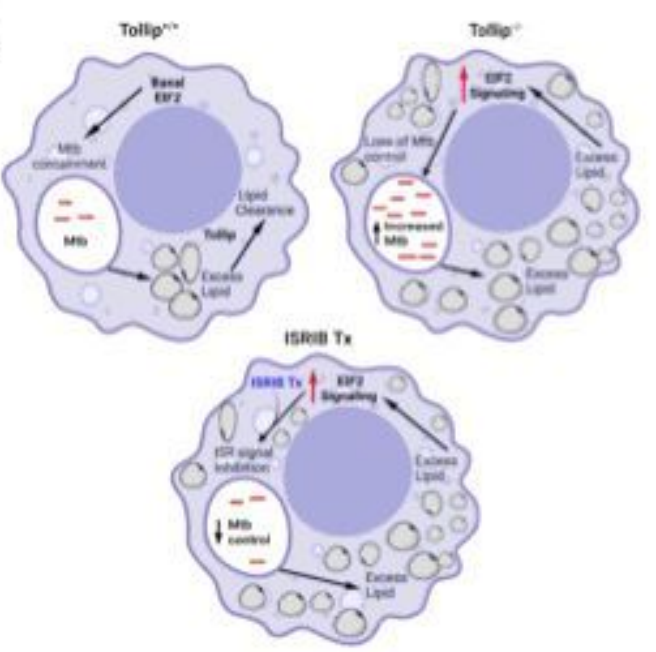

Figure 6

ISRIB treatment restores immune control in Tollip ${ }^{-/}$mice and improves host defense in B6 mice. 
A) B6 PEM (black circles) were treated with vehicle control or the EIF2 activator raphin-1 (black and white circles; $10 \mu \mathrm{M}$ ), infected with Mtb H37Rv expressing luxCDABE luminescence gene from Vibrio harleyi (Mtb-lux; MOI 1) and luminescence was measured over time. Error bars - SD. * $p<0.05 ; 2$-way ANOVA for an effect of raphin-1 controlling for time. $N=6$, experiment was performed three times.

B) Tollip ${ }^{-1}$ PEM were treated with vehicle control, MA, EIF2 inhibitor ISRIB (200nM), or both, then infected with Mtb-lux (MOI 1). Luminescence was measured 24 and 48 hours after infection. * $p<0.05,{ }^{\star *} p<0.01$, $\star \star \star * p<0.001,{ }^{* \star \star *} p<0.0001,2$-way ANOVA accounting for time and drug treatment. $N=6$, experiment was performed three times.

C) Experimental design. B6 and Tollip ${ }^{-/-}$mice were infected with 50-100 CFU Mtb and 15 days after infection, vehicle control, ISRIB ( $1 \mathrm{mg} / \mathrm{kg} /$ day), or raphin-1 $(1 \mathrm{mg} / \mathrm{kg} /$ day) were instilled intraperitoneally. Eight weeks after infection, mice were euthanized, bacterial CFU was measured, and tissue was collected for pathology analysis.

D-E) Mtb CFU in D) lungs and E) spleen from mice treated with ISRIB.

F-G) Mtb CFU in F) lungs and G) spleens from mice treated with raphin-1.

H) Representative images of lung (5x magnification) stained with H\&E from B6 and Tollip ${ }^{-/}$mice, with and without ISRIB treatment.

I) Number of nucleated cells per 20x high-powered field in Mtb-infected mice after ISRIB and raphin-1

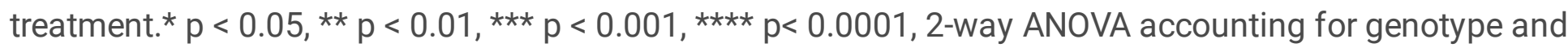
drug treatment. $\mathrm{N}=11$, experiment was performed twice.

J) Model of TOLLIP function and EIF2 activation during Mtb infection. During chronic Mtb infection, lipid products are released in Mtb-infected AM. TOLLIP prevents lipid accumulation, which contributes to ongoing host control of Mtb and maintains EIF2 signaling at a basal level. The resulting homeostasis of host-Mtb interaction results in ongoing host control. In chronically Mtb-infected Tollip ${ }^{-/} \mathrm{AM}$, the absence of TOLLIP permits ongoing lipid accumulation, which activates EIF2. EIF2 signals create conditions acceptable for increased bacterial burden. As a result, foam cell formation prevents Mtb killing and permits ongoing Mtb expansion. ISRIB inhibition restores Mtb control overall, including in the resistant B6 mouse, making it an effective host-directed therapeutic across genetic backgrounds.

\section{Supplementary Files}

This is a list of supplementary files associated with this preprint. Click to download.

- Tables2.csv

- Supplemental021422.docx 